\title{
Dietary Supplementation with Dried Syzygium cumini Flesh Improved Growth and Physiological Parameters in Hybrid \\ Catfish (Clarias macrocephalus $\times$ C. gariepinus)
}

\author{
Phukphon Munglue*, Kajita Matchima, Khwanduean Rattana, \\ Supavee Sangchanjiradet, and Kajohnpong Dasri
}

Faculty of Science, Ubon Ratchathani Rajabhat University, Ubon Ratchathani, 34000, Thailand

*Corresponding author.E-mail: phukphon.m@ubru.ac.th https://doi.org/10.12982/CMUJNS.2020.0046

Received: October 10, 2019

Revised: November 29, 2019

Accepted: December 12, 2019

\begin{abstract}
This research was aimed to evaluate the effects of dietary supplementation with dried S. cumini flesh (DSCF) on growth and physiological parameters in hybrid catfish (Clarias macrocephalus $\times C$. gariepinus). Fish (initial weight of $14.00 \pm 1.00 \mathrm{~g}$ ) were divided into four groups and fed with the diets containing DSCF at 0 (control), 1,3 and $5 \% / \mathrm{kg}$ diet for 8 weeks. After the experimental period, final weight, final length, weight gain, specific growth rate, average daily gain, and feed conversion efficiency were markedly increased, whereas feed conversion ratio was significantly decreased in the treated fish compared with the control fish $(P<0.05)$. The condition factor and survival rate did not differ significantly among the treatments $(P>0.05)$. Intestinal morphology was significantly improved in fish fed with DSCF containing diets $(P<0.05)$. No changes in white blood cell, red blood cell, hemoglobin, and hematocrit were observed between the treatments $(P>0.05)$. Meanwhile, the platelet was significantly increased in the tested fish compared with the control fish $(P<0.05)$. Aspartate aminotransferase, albumin, total protein, creatinine, uric acid, and triglyceride levels did not differ significantly between the treatments $(P>0.05)$. Serum glucose in fish fed with DSCF diet was significantly increased, while serum alkaline phosphatase was significantly lowered in the experimental groups compared to the control group $(P<0.05)$. Overall, these results indicate that the usefulness of DSCF as a phytogenic additive in aquafeeds with an effective level rage from 2.60 to $2.72 \% / \mathrm{kg}$ diet.
\end{abstract}

Keywords: Syzygium cumini, Hybrid catfish, Growth, Intestinal morphology, Hematology, Blood biochemistry 


\section{INTRODUCTION}

The global demand for aquaculture product consumption is constantly increased annually due to the growing population, dietary preferences, and income levels (FAO, 2012). Many challenges have been elucidated to bridge the gap between great demand and supply for fish products and the available report shows that the expansion of overfishing would have resulted in a decline in the fish population both in coastal and inland areas (Gupta, 2006).

From this existence, many fish species are encouraged to cultivate in several aquaculture practices (Bunting, 2013). Both semi-intensive and intensive aquaculture productions, for example, are kinds of production systems that commonly perform to achieve higher productivity per unit area (Wedemeyer, 1996). However, the intensive production system can cause stress and health problems in fish, resulting in reduced growth rate, increased pathogen infections, and decreased nutrient metabolism and utilization efficiency (Wedemeyer, 1996; Reverter et al., 2014). Chemicals and their derivatives have long been used in aquaculture industries to improve somatic growth and physiological parameters in fish (Awad and Awaad, 2017; Gabriel et al., 2019). Some reports have been pointed out that improper use of synthetic drugs may produce harmful effects both on consumer health and on the environments where they accumulate (Gabriel et al., 2015; Aanyu et al., 2018).

In recent years, most attention has been examined the applications of phytogenic additives to the fish diets due to their benefits such as lower toxicity, improvement of growth, reducing diseases and infections, and ecofriendly to environments (Villasante et al., 2016; Abdel-Tawwab et al., 2018; Munglue et al., 2019). It is well known that several plants have been intensively evaluated and developed to new drugs for use in the treatment of various diseases (Evans et al., 2002). From recent literature, plants have introduced to aquaculture production to improve growth performance, feed intake, and immune stimulation because of the actions of their phytochemicals including alkaloids, flavonoids, terpenoids, essential oils, and phenolic compounds (Reverter et al., 2014; Awad and Awaad, 2017; Munglue et al., 2019). However, current knowledge on the role of plants in modulating fish growth and health remains controversial (Mohammadi et al., 2018).

Syzygium cumini (Myrtaceae) is a large evergreen tree that is distributed in tropical and subtropical regions. Several parts of this plant have been used as traditional medicines in developing countries (Ayyanar and Subash-Babu, 2012). In folk medicine, it is effective in the treatment of diabetes, inflammation, cardiovascular diseases, gastrointestinal ulcers, cancer, and diarrhea (Mohamed et al., 2013). Additionally, anti-inflammatory, antioxidant, antiviral, antibacterial, antifungal, antiproliferative, and gastroprotective effects of $S$. cumini have been elucidated (Aqil et al., 2012; Ayyanar and Subash-Babu, 2012). The plant is showing the presence of anthocyanins, delphinidin-3-gentiobioside, malvidin-3- 
laminaribioside, petunidin-3-gentiobioside, gallic acid, cyanidin, mallic acid, diglycoside, petunidin, citric acid, glucose, raffinose, and fructose (Baliga et al., 2011; Ayyanar and Subash-Babu, 2012). It was documented that $S$. cumini flesh is rich in flavonoids, carotenoids, phenolic compounds, anthocyanins, minerals, and vitamins, which may provide novel compounds for natural food colorants in food industries and pharmaceutical products (Baliga et al., 2011; Aqil et al., 2012).

Recently, it has been reported that Pacific white leg shrimp (Litopenaeus vannamei) fed the diets supplemented with $S$. cumini leaf powder at $1 \%$ for 28 days potentially improved growth and non-specific immune parameters as well as the protection against Vibrio parahaemolyticus (Prabu et al., 2018). This scientific evidence would lead to support $S$. cumini as a potential phytogenic additive in aquatic diets. However, there were no reports on the growthpromoting property of $S$. cumini flesh in fish. For this important consideration, this research was aimed to evaluate the effects of dried S. cumini flesh (DSCF) supplementation on growth, intestinal morphology, hematology, and blood biochemistry of hybrid catfish (Clarias macrocephalus $\times$ C. gariepinus).

\section{MATERIALS AND METHODS}

\section{Plant materials and preparations}

Ripe fruits of S. cumini were collected from Warinchumrap District, Ubon Ratchathani, Thailand. They were cleaned thoroughly using distilled water and their seeds removed from the flesh. The samples of seedless fruits were dried in a hot air oven at $60^{\circ} \mathrm{C}$ until dry, grounded to fine powder, kept in plastic bags, and stored at $4^{\circ} \mathrm{C}$ until use.

\section{Diet samples and preparations}

A commercial basal diet (Centaco 3134) for catfish fingerlings containing $32 \%$ protein, $45 \%$ carbohydrate, $6 \%$ fiber, and $4 \%$ lipid was obtained from Centaco Group of Companies, Thailand and this protein level is recommended for a nutrient in the diet of catfish (Robinson et al., 2001). The diets were mixed with dried S. cumini flesh power at different levels of 0 (control), 1,3, and 5\%/kg diet. All diets samples and the basal diet were then coated with white egg, dried in a hot air oven at $40^{\circ} \mathrm{C}$ for $48 \mathrm{~h}$. Each formulated diet was kept in labeled zip-lock plastic bags and stored at $4^{\circ} \mathrm{C}$ for further use.

\section{Fish rearing and experimental design}

The experimental procedures were performed as the guidelines for the care and use of laboratory animals provided by the National Research Council of Thailand and Ubon Ratchathani Rajabhat University, Thailand.

Fingerlings of hybrid catfish were obtained from Ubon Ratchathani Fishery Cooperative, transported in polyethylene bags enriched with oxygen to 
the experimental site, and maintained in circular concrete tanks $(40 \mathrm{~cm}$ height and $70 \mathrm{~cm}$ width) containing $220 \mathrm{~L}$ dechlorinated water. During the acclimatization period for 2 weeks, the fish were observed for their health status daily. The fish (initial weight of $14.00 \pm 1.00 \mathrm{~g}$ ) were then randomly distributed into four treatments with three replicates (22 fish per replicates) and fed with the diets supplemented with DSCF at the levels of 0 (control), 1, 3, and $5 \% / \mathrm{kg}$ diet, ad libitum, twice a day, daily, for 8 weeks. Water qualities were maintained in the standard criteria for catfish cultivation (dissolved oxygen $8.00 \pm 1.00 \mathrm{mg} / \mathrm{L}$ and $\mathrm{pH} 7.50 \pm 0.40$ ). Half of the water was changed daily using the siphon. Fish dead was observed and recorded daily.

\section{Growth parameters and survival rate}

At the end of the experimental period, four fish from each replicate were randomly collected to evaluate growth and survival rate by using the following formulae (Gabriel et al., 2019):

Weight gain $(\mathrm{WG})(\mathrm{g})=$ final weight $(\mathrm{g})$ - initial weight $(\mathrm{g})$;

Specific growth rate $(\mathrm{SGR})(\% \mathrm{~g} /$ day $)=100 \times([$ In final weight $(\mathrm{g})-$ In initial weight $(\mathrm{g})] /$ the experimental period (days));

Average daily gain $(\mathrm{ADG})(\mathrm{g} /$ day $)=[$ final weight $(\mathrm{g})-$ initial weight $(\mathrm{g})] /$ the experimental period (days);

Feed conversion ratio $(\mathrm{FCR})=$ feed consumed by fish $(\mathrm{g}) / \mathrm{WG}(\mathrm{g})$;

Feed conversion efficiency $(\mathrm{FCE})=\mathrm{WG}(\mathrm{g}) /$ feed consumed by fish $(\mathrm{g})$;

Condition factor $(\mathrm{CF})\left(\mathrm{g} / \mathrm{cm}^{3}\right)=100 \times\left(\right.$ final weight $(\mathrm{g}) /$ length $\left.(\mathrm{cm})^{3}\right)$;

Survival rate $(\mathrm{SR})(\%)=100 \times($ initial number of fish/final number of fish $)$.

\section{Organosomatic indices}

At the end of the experimental trial, four fish from each replicate were collected, weighed, and anesthetized using $5 \mathrm{ppm}$ clove oil (Munglue et al., 2019). The abdomen was opened and internal organs including gill, heart, liver, intestine, stomach, kidney, and spleen were carefully removed, cleared from the abdominal fats and connective tissues, and weighed using a digital balance. The relative internal organ weight was calculated using the following equation:

The relative organ weight $(\%)=100 \times($ organ weight $(\mathrm{g}) /$ fish weight $(\mathrm{g}))$.

\section{Intestinal histology}

The intestines obtained from the experimental fish were divided into the proximal, middle, and distal portions. The samples were cleaned using $0.90 \%$ normal saline solution, fixed in $10 \%$ neutral buffer formalin, dehydrated in graded ethanol, equilibrated in xylene, embedded in paraffin wax, and cut into $5 \mu \mathrm{m}$. The sections were mounted on the glass slide and stained with hematoxylin and eosin $(\mathrm{H} \& \mathrm{E})$. Macromorphology and micromorphology of the intestines were determined under a light microscope connected with a computer running Dino 
Capture 2.0 software and interpreted as the standard guidelines (Baeverfjord and Krogdahl, 1996; Lazo et al., 2011; Munglue et al., 2019).

\section{Hematological indices}

After $24 \mathrm{~h}$ of the fasting period, four fish from each replicate were randomly harvested and anesthetized using clove oil $(5 \mathrm{ppm})$ in order to collect the blood from the caudal vein using a hypodermic syringe. Blood samples were transferred to a tube containing $\mathrm{K}_{2}$ EDTA. Hematological parameters including white blood cell (WBC), red blood cell (RBC), hematocrit (Hct), hemoglobin $(\mathrm{Hb})$, and platelet (PLT) were determined using the standard methods (Campbell and Ellis, 2007).

\section{Blood biochemistry profile}

Fish sera were collected from four fish in each replicate. Aspartate aminotransferase (AST), alkaline phosphatase (ALP), albumin, creatinine, uric acid, glucose, total protein, and triglyceride were evaluated by using the biochemical diagnostic kits obtained from Erba Lachema s.r.o., Czech Republic. The experimental procedures were performed according to the instructions provided by the manufacturer.

\section{Statistical analysis}

In this present study, completely randomized design was used. Throughout, results are presented as mean \pm SEM. Statistical significant difference among the treatments was evaluated using one way ANOVA followed by Duncan's multiple range tests. The significant differences were accepted when $P<0.05$. To estimate the optimal level of DSCF for application to the fish diet, the second-order polynomial regression model was used (Gabriel et al., 2019).

\section{RESULTS}

\section{Growth parameters and survival rate}

At the end of the feeding trial, fish that were fed with different DSCF diets showed significant increases in the final weight, final length, WG, SGR, ADG, and FCE compared with the control $(P<0.05)$. Significant decreases in FCR values were also obtained in fish fed the DSCF diets $(P<0.05)$. Condition factor and SR did not differ significantly among the treatments $(P>0.05)$ (Table 1). According to the second-order polynomial regression analysis on FW $\left(\mathrm{y}=-2.541 \mathrm{x}^{2}+13.366 \mathrm{x}, R^{2}=0.273, \mathrm{p}=0.005\right), \mathrm{WG}\left(\mathrm{y}=-2.565 \mathrm{x}^{2}+13.338 \mathrm{x}\right.$, $\left.R^{2}=0.305, \mathrm{p}=0.002\right)$, FCR $\left(\mathrm{y}=-0.181 \mathrm{x}^{2}+0.981 \mathrm{x}, R^{2}=0.304, \mathrm{p}=0.003\right)$, and FCE $\left(\mathrm{y}=-0.051 \mathrm{x}^{2}+0.267 \mathrm{x}, R^{2}=0.305, \mathrm{p}=0.002\right)$, the optimal level of DSCF was found to be between 2.60 and $2.71 \% / \mathrm{kg}$ diet, as shown in Figure 1. 
Table 1. Effects of different DSCF diets on growth parameters and survival rate of hybrid catfish.

\begin{tabular}{lllll}
\hline & \multicolumn{3}{c}{ DSCF (\%/kg diet) } \\
\cline { 2 - 5 } & $\mathbf{0}$ & $\mathbf{1}$ & $\mathbf{5}$ \\
\hline IW (g) & $14.55 \pm 0.58$ & $14.22 \pm 0.61$ & $14.88 \pm 0.69$ & $15.11 \pm 0.26$ \\
FW (g) & $33.55 \pm 2.39^{\mathrm{c}}$ & $47.00 \pm 3.39^{\mathrm{a}}$ & $50.55 \pm 6.09^{\mathrm{a}}$ & $38.11 \pm 2.13^{\mathrm{b}}$ \\
IL (cm) & $11.83 \pm 0.26$ & $11.78 \pm 0.32$ & $11.89 \pm 0.25$ & $12.22 \pm 0.22$ \\
FL (cm) & $16.67 \pm 0.03^{\mathrm{b}}$ & $18.33 \pm 0.07^{\mathrm{a}}$ & $18.77 \pm 0.09^{\mathrm{a}}$ & $17.28 \pm 0.06^{\mathrm{b}}$ \\
WG (g) & $19.00 \pm 1.12^{\mathrm{c}}$ & $32.77 \pm 1.27^{\mathrm{a}}$ & $35.66 \pm 1.52^{\mathrm{a}}$ & $23.00 \pm 1.17^{\mathrm{b}}$ \\
SGR (\%/day) & $1.39 \pm 0.03^{\mathrm{c}}$ & $2.08 \pm 0.05^{\mathrm{a}}$ & $2.15 \pm 0.02^{\mathrm{a}}$ & $1.49 \pm 0.04^{\mathrm{b}}$ \\
ADG (g/day) & $0.33 \pm 0.03^{\mathrm{c}}$ & $0.58 \pm 0.05^{\mathrm{a}}$ & $0.63 \pm 0.09^{\mathrm{a}}$ & $0.41 \pm 0.03^{\mathrm{b}}$ \\
FCR & $2.93 \pm 0.04^{\mathrm{a}}$ & $1.64 \pm 0.05^{\mathrm{c}}$ & $1.66 \pm 0.04^{\mathrm{c}}$ & $2.32 \pm 0.02^{\mathrm{b}}$ \\
FCE & $0.36 \pm 0.02^{\mathrm{c}}$ & $0.65 \pm 0.06^{\mathrm{a}}$ & $0.71 \pm 0.01^{\mathrm{a}}$ & $0.46 \pm 0.03^{\mathrm{b}}$ \\
CF (g/cm $\left.{ }^{3}\right)$ & $0.70 \pm 0.03$ & $0.76 \pm 0.02$ & $0.74 \pm 0.02$ & $0.74 \pm 0.02$ \\
SR (\%) & $100 \pm 0.00$ & $99.17 \pm 0.83$ & $100 \pm 0.00$ & $99.17 \pm 0.83$ \\
\hline
\end{tabular}

Note: In each row, different superscripts ${ }^{(\mathrm{a}-\mathrm{c})}$ represent significant differences at $P<0.05$. Data are Mean \pm SEM; $\mathrm{n}=4 ; \mathrm{IW}=$ initial weight $(\mathrm{g}) ; \mathrm{FW}=$ final weight $(\mathrm{g}) ; \mathrm{IL}=$ initial length $(\mathrm{cm}) ; \mathrm{FL}=$ final length $(\mathrm{cm})$; $\mathrm{WG}=$ weight gain $(\mathrm{g}) ; \mathrm{SGR}=$ specific growth rate $(\% /$ day $) ; \quad \mathrm{ADG}=$ average daily gain $(\mathrm{g} / \mathrm{day}) ;$ $\mathrm{FCR}=$ feed conversion ratio; $\mathrm{FCE}=$ feed conversion efficiency; $\mathrm{CF}=$ condition factor $\left(\mathrm{g} / \mathrm{cm}^{3}\right)$; $\mathrm{SR}=$ survival rate $(\%)$. 

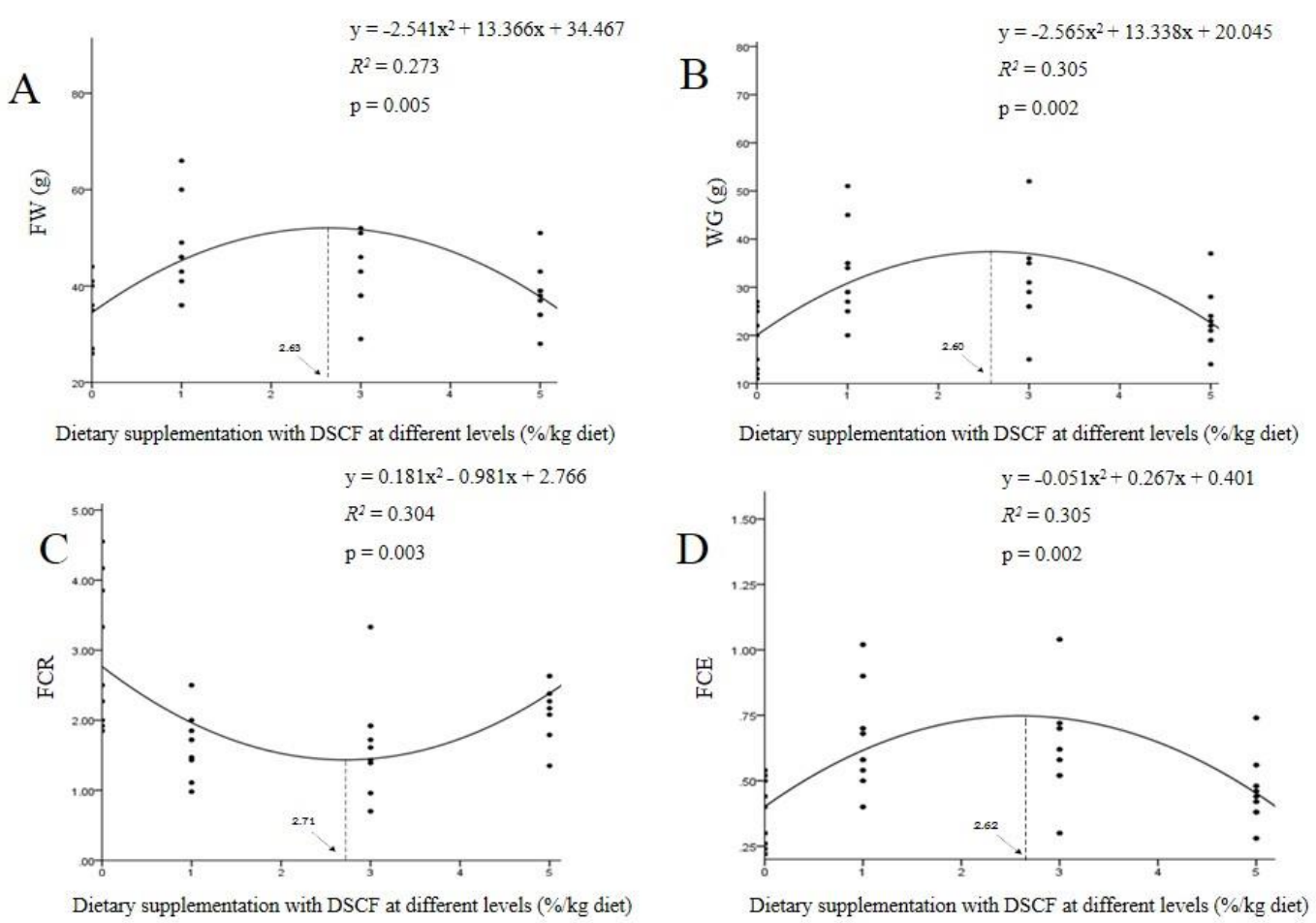

Figure 1. The second-order polynomial regression analysis on (A) final weight (FW), (B) weight gain (WG), (C) feed conversion ratio (FCR), and (D) feed conversion efficiency (FCE) of hybrid catfish fed different DSCF diets for 8 weeks.

\section{Organosomatic indices}

As shown in Table 2, DSCF supplementation caused a significant increase in the relative organ weights of the heart, intestine, stomach, kidney, and spleen compared with the basal diet $(P<0.05)$. No significant differences were observed in the relative organ weights of the gill and liver among the groups $(P>0.05)$.

Table 2. Effects of different DSCF diets on organosomatic indices of hybrid catfish.

\begin{tabular}{lllll}
\hline & \multicolumn{4}{c}{ DSCF (\%/kg diet) } \\
\cline { 2 - 5 } & $\mathbf{0}$ & $\mathbf{1}$ & $\mathbf{3}$ & $\mathbf{5}$ \\
\hline Gill (\%) & $14.67 \pm 0.78$ & $14.78 \pm 1.12$ & $14.56 \pm 0.82$ & $16.33 \pm 0.60$ \\
Heart (\%) & $32.78 \pm 2.80^{\mathrm{c}}$ & $47.33 \pm 3.27^{\mathrm{a}}$ & $50.56 \pm 6.10^{\mathrm{a}}$ & $38.11 \pm 2.14^{\mathrm{b}}$ \\
Liver (\%) & $11.83 \pm 0.26$ & $11.78 \pm 0.32$ & $11.89 \pm 0.25$ & $12.22 \pm 0.22$ \\
Intestine (\%) & $16.67 \pm 0.43^{\mathrm{b}}$ & $18.33 \pm 0.37^{\mathrm{a}}$ & $18.77 \pm 0.79^{\mathrm{a}}$ & $17.28 \pm 0.36^{\mathrm{ab}}$ \\
Stomach (\%) & $20.44 \pm 2.38^{\mathrm{b}}$ & $35.22 \pm 2.67^{\mathrm{a}}$ & $36.22 \pm 5.33^{\mathrm{a}}$ & $21.78 \pm 1.55^{\mathrm{b}}$ \\
Kidney (\%) & $1.40 \pm 0.10^{\mathrm{b}}$ & $2.09 \pm 0.17^{\mathrm{a}}$ & $2.15 \pm 0.11^{\mathrm{a}}$ & $1.50 \pm 0.04^{\mathrm{b}}$ \\
Spleen (\%) & $0.37 \pm 0.04^{\mathrm{b}}$ & $0.63 \pm 0.05^{\mathrm{a}}$ & $0.65 \pm 0.10^{\mathrm{a}}$ & $0.39 \pm 0.03^{\mathrm{b}}$ \\
\hline
\end{tabular}

Note: In each row, different superscripts ${ }^{(\mathrm{a}-\mathrm{c})}$ represent significant differences at $P<0.05$. Data are Mean \pm SEM; $\mathrm{n}=4$. 


\section{Intestinal macromorphology}

The effects of different DSCF diets on intestinal macromorphology of hybrid catfish are summarized in Table 3.

Table 3. Effects of different DSCF diets on intestinal macromorphology of hybrid catfish.

\begin{tabular}{lllll}
\hline & \multicolumn{4}{c}{ DSCF (\%/kg diet) } \\
\cline { 2 - 5 } Proximal intestine & $\mathbf{1}$ & $\mathbf{3}$ & $\mathbf{5}$ \\
hV $(\mu \mathrm{m})$ & $1916.94 \pm 15.94^{\mathrm{b}}$ & $2123.48 \pm 10.22^{\mathrm{a}}$ & $1609.47 \pm 65.65^{\mathrm{b}}$ & $1813.21 \pm 12.42^{\mathrm{b}}$ \\
wV $(\mu \mathrm{m})$ & $296.97 \pm 18.66^{\mathrm{a}}$ & $308.52 \pm 26.88^{\mathrm{a}}$ & $194.00 \pm 13.17^{\mathrm{c}}$ & $190.93 \pm 10.97^{\mathrm{c}}$ \\
tML $(\mu \mathrm{m})$ & $272.19 \pm 21.91^{\mathrm{a}}$ & $221.03 \pm 10.69^{\mathrm{b}}$ & $202.52 \pm 18.22^{\mathrm{c}}$ & $187.82 \pm 15.19^{\mathrm{c}}$ \\
tICML $(\mu \mathrm{m})$ & $181.53 \pm 7.24^{\mathrm{a}}$ & $152.20 \pm 9.93^{\mathrm{ab}}$ & $146.63 \pm 1.83^{\mathrm{b}}$ & $130.85 \pm 1.36^{\mathrm{c}}$ \\
tOLML $(\mu \mathrm{m})$ & $88.29 \pm 6.19^{\mathrm{a}}$ & $72.17 \pm 4.87^{\mathrm{b}}$ & $52.62 \pm 5.13^{\mathrm{c}}$ & $55.70 \pm 4.63^{\mathrm{c}}$ \\
wLP $(\mu \mathrm{m})$ & $27.00 \pm 4.11$ & $42.09 \pm 7.91$ & $38.93 \pm 7.50$ & $24.00 \pm 6.54$ \\
\hline Middle intestine & & & & \\
hV $(\mu \mathrm{m})$ & $1019.38 \pm 26.35^{\mathrm{c}}$ & $1583.46 \pm 10.00^{\mathrm{a}}$ & $1324.23 \pm 95.38^{\mathrm{b}}$ & $1349.82 \pm 16.30^{\mathrm{b}}$ \\
wV $(\mu \mathrm{m})$ & $331.67 \pm 20.31^{\mathrm{a}}$ & $245.07 \pm 18.39^{\mathrm{b}}$ & $170.64 \pm 11.34^{\mathrm{c}}$ & $210.80 \pm 12.70^{\mathrm{b}}$ \\
tML $(\mu \mathrm{m})$ & $182.38 \pm 25.98$ & $173.90 \pm 17.22$ & $197.13 \pm 17.21$ & $148.74 \pm 14.84$ \\
tICML $(\mu \mathrm{m})$ & $122.23 \pm 20.86$ & $112.39 \pm 14.39$ & $115.44 \pm 10.74$ & $94.58 \pm 9.46$ \\
tOLML $(\mu \mathrm{m})$ & $53.37 \pm 6.38^{\mathrm{b}}$ & $50.28 \pm 3.95^{\mathrm{b}}$ & $71.12 \pm 5.30^{\mathrm{a}}$ & $52.02 \pm 7.69^{\mathrm{b}}$ \\
wLP $(\mu \mathrm{m})$ & $39.40 \pm 2.43$ & $38.58 \pm 9.00$ & $41.37 \pm 5.24$ & $32.56 \pm 4.00$ \\
\hline Distal intestine & & & & \\
hV $(\mu \mathrm{m})$ & $934.98 \pm 82.43$ & $1110.70 \pm 53.78$ & $895.64 \pm 49.12$ & $926.21 \pm 92.96$ \\
wV $(\mu \mathrm{m})$ & $268.39 \pm 17.70^{\mathrm{a}}$ & $267.62 \pm 15.78^{\mathrm{a}}$ & $177.06 \pm 14.68^{\mathrm{b}}$ & $234.49 \pm 16.71^{\mathrm{a}}$ \\
tML $(\mu \mathrm{m})$ & $173.99 \pm 15.58^{\mathrm{b}}$ & $145.55 \pm 12.88^{\mathrm{b}}$ & $133.96 \pm 10.49^{\mathrm{c}}$ & $240.06 \pm 36.65^{\mathrm{a}}$ \\
tICML $(\mu \mathrm{m})$ & $114.20 \pm 13.22^{\mathrm{b}}$ & $89.40 \pm 7.84^{\mathrm{c}}$ & $86.56 \pm 6.76^{\mathrm{c}}$ & $165.07 \pm 26.43^{\mathrm{a}}$ \\
tOLML $(\mu \mathrm{m})$ & $52.78 \pm 3.99^{\mathrm{b}}$ & $49.20 \pm 5.81^{\mathrm{b}}$ & $48.52 \pm 3.64^{\mathrm{b}}$ & $72.28 \pm 10.69^{\mathrm{a}}$ \\
wLP $(\mu \mathrm{m})$ & $50.17 \pm 5.03^{\mathrm{a}}$ & $51.28 \pm 6.76^{\mathrm{a}}$ & $31.53 \pm 4.55^{\mathrm{b}}$ & $45.94 \pm 10.54^{\mathrm{ab}}$ \\
\hline & & & & \\
\hline
\end{tabular}

Note: In each row, different superscripts ${ }^{(a-c)}$ represent significant differences $(\mathrm{p}<0.05)$. Data are Mean \pm SEM; $\mathrm{n}=4 ; \mathrm{hV}=$ villi height $(\mu \mathrm{m}) ; \mathrm{wV}=$ villi width $(\mu \mathrm{m}) ; \mathrm{tML}=$ total muscular layer $(\mu \mathrm{m}) ; \mathrm{tCML}=$ total inner circular muscle layer $(\mu \mathrm{m})$; tOLML = total outer longitudinal muscle layer $(\mu \mathrm{m}) ; \mathrm{wLP}=$ lamina propria width $(\mu \mathrm{m})$.

In the proximal intestines, fish that were fed with DSCF containing diets showed significant increases in villi heights compared with the control $(P<0.05)$. Meanwhile, significant decreases in villi widths, total muscular layers, total inner circulatory muscle layers, and outer longitudinal muscle layers were detected in the tested fish $(P<0.05)$. However, there were no significant changes in the lamina propria widths among the diets $(P>0.05)$. 
In the middle intestine, villi heights and total outer longitudinal muscle layers were found to be significantly decreased in fish fed the DSCF diets compared with the control $(P<0.05)$. In contrast, villi widths in the experimental fish were significantly reduced $(P<0.05)$. Total muscular layers, total inner circular muscle layers, and lamina propria widths were similar among the treatments $(P>0.05)$.

In the distal intestine, dietary supplementation of 3\%DSCF produced significant decreases in villi width, total muscular layer, total inner circulatory muscle layer, and lamina propria width compared with the control $(P<0.05)$. On the other hand, total muscular layer, total inner circulatory muscle layer, and total outer longitudinal muscle layer were significantly increased in fish fed the 5\% DSCF diet compared with fish fed the control diet $(P<0.05)$. The results also showed that there were no significant differences in villi heights between the treatments $(P>0.05)$.

\section{Intestinal micromorphology}

The effects of DSCF-based diets on intestinal micromorphology of hybrid catfish are presented in Table 4.

In the proximal intestine, supplementation with $1 \% \mathrm{DSCF}$ significantly increased enterocyte height and supranucleus height compared with the control $(P<0.05)$. Fish that were fed with 3 and 5\%DSCF diets showed significant decreases in enterocyte heights, supranucleus heights, and subnucleus heights compared with the control $(P<0.05)$, while microvilli height increased significantly in fish fed 5\%DSCF containing diet $(P<0.05)$. Significant decreases in nucleus widths were noticed in the experimental fish in comparison with the control fish $(P<0.05)$. However, there were no changes in nucleus heights among the treatments $(P>0.05)$.

In the middle intestine, enterocyte height, supranucleus height, and subnucleus height were significantly enhanced in fish fed with $1 \%$ DSCF diet $(P<0.05)$; meanwhile fish that were fed with $3 \% \mathrm{DSCF}$ diet showed significant decreases in enterocyte height, supranucleus height, and subnucleus height compared with the control $(P<0.05)$. Significant decrease in the nucleus height was found in fish fed 5\%DSCF added diet $(P<0.05)$. Additionally, microvilli height was significantly enhanced by $3 \%$ DSCF diet $(P<0.05)$. Nucleus widths of the treated fish were significantly higher than those of the control fish $(P<0.05)$.

In the distal intestine, fish that were fed with the diets containing 3 and $5 \% \mathrm{DSCF}$ showed significant increases in the microvilli heights compared with the control $(P<0.05)$. Meanwhile, supranucleus heights were significantly decreased in dietary DSCF $(P<0.05)$. Nucleus heights and nucleus widths in fish fed the diets containing DSCF were significantly increased $(P<0.05)$. Enterocyte heights and subnucleus heights of the experimental groups did not differ when compared with the control group $(P>0.05)$. 
Table 4. Effects of different DSCF diets on intestinal micromorphology of hybrid catfish.

\begin{tabular}{lllll}
\hline & \multicolumn{4}{c}{ DSCF (\%/kg diet) } \\
\cline { 2 - 5 } Proximal intestine & $\mathbf{0}$ & $\mathbf{3}$ & $\mathbf{5}$ \\
$\mathrm{hMV}(\mu \mathrm{m})$ & $3.36 \pm 0.22^{\mathrm{b}}$ & $2.82 \pm 0.26^{\mathrm{b}}$ & $2.79 \pm 0.44^{\mathrm{b}}$ & $4.85 \pm 0.23^{\mathrm{a}}$ \\
$\mathrm{hE}(\mu \mathrm{m})$ & $77.08 \pm 7.31^{\mathrm{b}}$ & $123.15 \pm 11.69^{\mathrm{a}}$ & $59.96 \pm 8.45^{\mathrm{c}}$ & $49.08 \pm 3.33^{\mathrm{c}}$ \\
$\mathrm{hSN}(\mu \mathrm{m})$ & $30.64 \pm 3.73^{\mathrm{b}}$ & $55.10 \pm 7.87^{\mathrm{a}}$ & $21.54 \pm 1.91^{\mathrm{c}}$ & $20.62 \pm 2.01^{\mathrm{c}}$ \\
$\mathrm{hBN}(\mu \mathrm{m})$ & $29.16 \pm 3.38^{\mathrm{a}}$ & $23.80 \pm 3.01^{\mathrm{ab}}$ & $20.80 \pm 5.98^{\mathrm{bc}}$ & $16.55 \pm 1.28^{\mathrm{c}}$ \\
$\mathrm{hN}(\mu \mathrm{m})$ & $11.13 \pm 0.26$ & $12.42 \pm 0.28$ & $11.89 \pm 0.98$ & $10.35 \pm 0.19$ \\
$\mathrm{wN}(\mu \mathrm{m})$ & $8.04 \pm 0.18^{\mathrm{a}}$ & $7.46 \pm 0.25^{\mathrm{b}}$ & $7.36 \pm 0.20^{\mathrm{b}}$ & $7.30 \pm 0.18^{\mathrm{b}}$ \\
\hline Middle intestine & & & & \\
$\mathrm{hMV}(\mu \mathrm{m})$ & $2.54 \pm 0.48^{\mathrm{b}}$ & $2.54 \pm 0.48^{\mathrm{b}}$ & $4.22 \pm 0.23^{\mathrm{a}}$ & $3.03 \pm 0.37^{\mathrm{b}}$ \\
$\mathrm{hE}(\mu \mathrm{m})$ & $76.21 \pm 4.08^{\mathrm{b}}$ & $112.57 \pm 21.17^{\mathrm{a}}$ & $48.02 \pm 3.72^{\mathrm{d}}$ & $60.07 \pm 4.25^{\mathrm{c}}$ \\
$\mathrm{hSN}(\mu \mathrm{m})$ & $30.70 \pm 1.80^{\mathrm{b}}$ & $45.67 \pm 1.99^{\mathrm{a}}$ & $20.42 \pm 2.75^{\mathrm{c}}$ & $27.09 \pm 2.76^{\mathrm{b}}$ \\
$\mathrm{hBN}(\mu \mathrm{m})$ & $23.93 \pm 2.52^{\mathrm{b}}$ & $35.08 \pm 5.17^{\mathrm{a}}$ & $14.72 \pm 2.13^{\mathrm{c}}$ & $19.36 \pm 1.61^{\mathrm{b}}$ \\
$\mathrm{hN}(\mu \mathrm{m})$ & $12.50 \pm 0.38^{\mathrm{a}}$ & $12.38 \pm 0.34^{\mathrm{a}}$ & $12.47 \pm 0.28^{\mathrm{a}}$ & $9.82 \pm 0.28^{\mathrm{b}}$ \\
$\mathrm{wN}(\mu \mathrm{m})$ & $6.74 \pm 0.22^{\mathrm{b}}$ & $7.54 \pm 0.25^{\mathrm{a}}$ & $7.36 \pm 0.20^{\mathrm{a}}$ & $7.52 \pm 0.13^{\mathrm{a}}$ \\
\hline Distal intestine & & & & \\
$\mathrm{hMV}(\mu \mathrm{m})$ & $2.23 \pm 0.29^{\mathrm{c}}$ & $2.33 \pm 0.05^{\mathrm{c}}$ & $3.15 \pm 0.27^{\mathrm{b}}$ & $4.78 \pm 0.32^{\mathrm{a}}$ \\
$\mathrm{hE}(\mu \mathrm{m})$ & $77.76 \pm 7.59$ & $85.01 \pm 7.35$ & $62.99 \pm 4.70$ & $72.73 \pm 6.00$ \\
$\mathrm{hSN}(\mu \mathrm{m})$ & $42.05 \pm 6.49^{\mathrm{a}}$ & $42.44 \pm 5.23^{\mathrm{a}}$ & $31.17 \pm 2.99^{\mathrm{b}}$ & $29.64 \pm 3.88^{\mathrm{b}}$ \\
$\mathrm{hBN}(\mu \mathrm{m})$ & $19.47 \pm 2.86^{\mathrm{ab}}$ & $21.71 \pm 2.44^{\mathrm{a}}$ & $16.14 \pm 1.79^{\mathrm{b}}$ & $24.38 \pm 2.72^{\mathrm{a}}$ \\
$\mathrm{hN}(\mu \mathrm{m})$ & $12.12 \pm 0.44^{\mathrm{c}}$ & $14.47 \pm 0.35^{\mathrm{ab}}$ & $13.37 \pm 0.46^{\mathrm{b}}$ & $14.59 \pm 0.43^{\mathrm{a}}$ \\
$\mathrm{wN}(\mu \mathrm{m})$ & $6.20 \pm 0.20^{\mathrm{b}}$ & $7.17 \pm 0.20^{\mathrm{a}}$ & $6.61 \pm 0.18^{\mathrm{b}}$ & $7.06 \pm 0.16^{\mathrm{a}}$ \\
\hline $\mathrm{N}$ & & & & \\
\hline
\end{tabular}

Note: In each row, different superscripts $\left.{ }^{\text {a-d }}\right)$ represent significant differences $(P<0.05)$; Data are Mean \pm SEM; $\mathrm{n}=4$; hMV; microvilli height $(\mu \mathrm{m}) ; \mathrm{hE}=$ enterocyte height $(\mu \mathrm{m}) ; \mathrm{hSN}=$ supranucleus height $(\mu \mathrm{m}) ; \mathrm{hBN}=$ subnucleus height $(\mu \mathrm{m}) ; \mathrm{hN}=$ nucleus height $(\mu \mathrm{m}) ; \mathrm{wN}=$ nucleus width $(\mu \mathrm{m})$.

\section{Hematological indices}

As shown in Table 5, fish that were fed with a mixture of DSCF at the concentration of 5\% significantly increased in WBC and platelet count compared with the control and fish fed with 1 and 3\%DSCF diets $(P<0.05)$. Evidently, RBC, $\mathrm{Hb}$, and Hct had a tendency to increase with increasing DSCF levels in the diets but these hematological values did not reach significantly different levels when compared with the control $(P>0.05)$. 
Table 5. Effects of different DSCF diets on hematological indices of hybrid catfish.

\begin{tabular}{|c|c|c|c|c|}
\hline & \multicolumn{4}{|c|}{ DSCF (\%/kg diet) } \\
\hline & $\mathbf{0}$ & 1 & 3 & 5 \\
\hline $\mathrm{WBC}\left(\times 10^{3}\right.$ cell $\left./ \mu \mathrm{L}\right)$ & $44.13 \pm 1.74^{\mathrm{b}}$ & $38.23 \pm 0.26^{\mathrm{b}}$ & $43.47 \pm 1.78^{b}$ & $51.53 \pm 4.23^{\mathrm{a}}$ \\
\hline $\mathrm{RBC}\left(\times 10^{6}\right.$ cell $\left./ \mu \mathrm{L}\right)$ & $2.23 \pm 0.24$ & $2.34 \pm 0.08$ & $2.53 \pm 0.15$ & $2.68 \pm 0.03$ \\
\hline $\mathrm{Hb}(\mathrm{g} / \mathrm{dL})$ & $10.30 \pm 1.00$ & $10.80 \pm 0.06$ & $11.13 \pm 0.57$ & $11.53 \pm 0.62$ \\
\hline $\operatorname{Hct}(\%)$ & $29.97 \pm 0.78$ & $30.07 \pm 0.47$ & $32.57 \pm 1.39$ & $32.13 \pm 1.71$ \\
\hline $\operatorname{PLT}\left(\times 10^{3}\right.$ cell $\left./ \mu \mathrm{L}\right)$ & $27.33 \pm 2.40^{\mathrm{b}}$ & $28.32 \pm 3.84^{\mathrm{b}}$ & $28.33 \pm 2.32^{b}$ & $41.00 \pm 8.50^{\mathrm{a}}$ \\
\hline
\end{tabular}

Note: In each row, different superscripts $\left({ }^{\mathrm{a}, \mathrm{b}}\right)$ represent significant differences $(P<0.05)$; Data are Mean \pm SEM; $\mathrm{n}=4 ; \mathrm{WBC}=$ white blood cell $\left(\times 10^{3}\right.$ cell $\left./ \mu \mathrm{L}\right) ; \mathrm{RBC}=$ red blood cell $\left(\times 10^{6}\right.$ cell $\left./ \mu \mathrm{L}\right) ; \mathrm{Hb}=$ hemoglobin $(\mathrm{g} / \mathrm{dL}) ; \mathrm{Hct}=$ hematocrit $(\%) ;$ PLT $=$ platelet $\left(\times 10^{3}\right.$ cell $\left./ \mu \mathrm{L}\right)$.

\section{Blood biochemistry profile}

As demonstrated in Table 6, dietary supplementation with 5\%DSCF produced a significant enhancement in the blood glucose level compared with the other diets $(P<0.05)$. ALP level was significantly reduced in fish fed 1\%DSCF diet $(P<0.05)$. However, DSCF containing diets did not significantly influence the levels of AST, albumin, total protein, creatinine, uric acid, and triglyceride when compared with the control $(P>0.05)$.

Table 6. Effects of different DSCF diets on blood biochemistry of hybrid catfish.

\begin{tabular}{lllll}
\hline & \multicolumn{4}{c}{ DSCF (\%/kg diet) } \\
\cline { 2 - 5 } & $\mathbf{0}$ & $\mathbf{3}$ & $\mathbf{3}$ \\
\hline AST (U/L) & $234.84 \pm 45.01$ & $265.86 \pm 26.61$ & $226.62 \pm 21.12$ & $320.93 \pm 27.06$ \\
ALP (U/L) & $27.62 \pm 8.66^{\mathrm{a}}$ & $17.63 \pm 1.63^{\mathrm{b}}$ & $25.27 \pm 4.24^{\mathrm{a}}$ & $27.04 \pm 6.14^{\mathrm{a}}$ \\
Albumin (mg/dL) & $1.56 \pm 0.16$ & $1.68 \pm 0.07$ & $1.49 \pm 0.05$ & $1.38 \pm 0.04$ \\
Total Protein (mg/dL) & $4.04 \pm 0.18$ & $3.57 \pm 0.11$ & $4.02 \pm 0.40$ & $3.68 \pm 0.15$ \\
Creatinine (mg/dL) & $0.96 \pm 0.44$ & $1.29 \pm 0.93$ & $1.96 \pm 1.27$ & $1.04 \pm 0.30$ \\
Uric Acid (mg/dL) & $2.12 \pm 0.27$ & $1.82 \pm 0.34$ & $1.81 \pm 0.60$ & $1.42 \pm 0.59$ \\
Glucose (mg/dL) & $168.33 \pm 12.22^{\mathrm{b}}$ & $142.14 \pm 20.72^{\mathrm{b}}$ & $148.10 \pm 30.74^{\mathrm{b}}$ & $264.76 \pm 17.06^{\mathrm{a}}$ \\
Triglyceride (mg/dL) & $176.93 \pm 47.58$ & $138.96 \pm 28.43$ & $134.60 \pm 21.53$ & $163.31 \pm 30.80$ \\
\hline
\end{tabular}

Note: In each row, different superscripts $\left({ }^{\mathrm{a}, \mathrm{b}}\right)$ represent significant differences $(P<0.05)$; Data are Mean \pm SEM; $\mathrm{n}=4 ; \mathrm{AST}=$ aspartate aminotransferase $(\mathrm{U} / \mathrm{L}) ; \mathrm{ALP}=$ alkaline phosphatase $(\mathrm{U} / \mathrm{L})$.

\section{DISCUSSION}

This research indicated for the first time that DSCF supplemented diets significantly improved growth, feed utilization, organosomatic indices, intestinal morphology, WBC, PLT, and serum glucose in hybrid catfish when compared with the control. Serum ALP was significantly declined in the treated fish compared with the control fish. However, there were no changes in CF, SR, RBC, 
$\mathrm{Hb}, \mathrm{Hct}, \mathrm{AST}$, albumin, total protein, creatinine, uric acid, and triglyceride among the groups. Therefore, this experiment confirms that DSCF could potentially be submitted as a phytogenic additive in aquafeeds.

After 8 weeks of the experimental period, it was found that growth parameters including final weight, final length, WG, SGR, ADG, and FCE of fish fed the diets supplemented with DSCF were significantly higher than those of fish fed the basal diet. Meanwhile, FCR values of fish fed 1 and 3\%DSCF diets were significantly decreased. Additionally, CF and SR did not change significantly among the groups. Growth-promoting properties of various plants in aquatic animals are well reported (Reverter et al., 2014; Panase et al., 2018). The previous report indicated that Pacific white leg shrimp fed the diet containing $S$. cumini leaf power at $1 \%$ for 28 days produced a significant enhancement of growth indices including WG, SGR, and protein efficiency ratio compared with the control (Prabu et al., 2018). Mohammadi et al. (2018) found that common carp (Cyprinus carpio) fed the diets containing 0, 0.5, 1, 2, and $4 \%$ date palm (Phoenix dactylifera) seed extract for 60 days showed a significant improvement in growth parameters and feed utilization efficiency. In this research, the underlying mechanisms of action of herbal plants on the growth of fish are not well understood. Such reports indicated that active compounds found in the plant extracts may potentially improve feed intake, nutrient digestion, metabolism, and absorption capacity of the nutrients by increasing the activities of certain intestinal digestive enzymes (Haghbayan and Mehrgan, 2015; Mohammadi et al., 2018; Sewaka et al., 2019). Furthermore, scientific studies have demonstrated that positive effects of diets supplemented with phytogenic additives would directly induce the expression of growth hormone $(\mathrm{GH})$ and insulin-like growth factors (IGFs) mRNA in order to promote muscle cell proliferation and differentiation, resulting in a pronounced increase in the growth rate of fish (Sruthi et al., 2018; Ahmadifar et al., 2019). Therefore, this present study implies that DSCF may be a useful somatic growth stimulant in farmed fish. By using the second-order polynomial analysis, the optimal concentration of DSCF was evaluated to be between 2.60 and $2.71 \% / \mathrm{kg}$ diet.

Observations on organosomatic indices could be useful for detecting the physiological responses of the tested fish that were fed with the diet containing phytogenic additive (Serrano et al., 2012). As noted, the results indicated that the relative weights of the heart, intestine, stomach, kidney, and spleen of fish fed the diets containing DSCF were significantly greater than those of fish fed the basal diet. Previous studies have revealed that increased organ weight is related to abiotic and biotic factors, which can cause an increase in hyperplasia and hypertrophy of the cell due to the improvement in cell metabolism or cell division (Dekić et al., 2016; Panase et al., 2018). The result of this study is in line with the report of Panase et al. (2018) who indicated that hybrid catfish that were fed with the diets supplemented with Euphorbia hirta leaf extract at the levels of 300, 500, and $800 \mathrm{mg} / \mathrm{kg}$ for 90 days showed significant increases in viscerosomatic index, 
hepatosomatic index, and intraperitoneal fat compared with the control. Therefore, it is possible to hypothesize that some active compounds found in DSCF may activate cell division and differentiation in internal organs, resulting in the enhancement of the relative organ weight observed in the study (Serrano et al., 2012; Panase et al., 2018).

Studies on intestinal histology could be useful for evaluating the effects of dietary supplemented with some phytogenic additives on the intestinal health status of fish (Escaffre et al., 2007; Ferrara et al., 2015). It has been postulated that increased intestinal villi height and submucosal width would provide a higher intestinal nutrient absorptive area for increased nutrient assimilation capacities of the gut, which subsequently led to the improvement in the growth of fish (Tiengtam et al., 2015; Munglue et al., 2019). It is well known that fish intestines consist of inner circular and outer longitudinal layers of smooth muscle. The prospective functions of muscular layers are 1) to produce a wavelike series of intestinal muscular contractions in order to aid the digestion and absorption of ingested food and; 2) to modulate osmoregulation (Lazo et al., 2011). In this study, DSCF supplementation resulted in the improvement of intestinal muscular thicknesses, which would help to support the utilization of nutrients and water balance in fish (Wilson and Castro, 2011). It was demonstrated that improved intestinal villi height, submucosal width, and muscular thickness would be related to positive effects of some phytochemicals on the cell cycle regulators to greatly increase intestinal cell proliferation and differentiation (Gupta and Sandhu, 1998; Villasante et al., 2016; Munglue et al., 2019). The lamina propria lines beneath the intestinal epithelial layer, which contains several components such as leukocytes, fibroblasts, loose connective tissues, vascular networks, and lymphatic ducts. It is well known that the functions of the lamina propria are to support and nourish the intestinal epithelium (Baeverfjord and Krogdahl, 1996). A widening of the lamina propria resembles conditions like intestinal inflammation and meal-induce pathology in fish (Baeverfjord and Krogdahl, 1996). In this present study, there were no changes in the lamina propria widths in fish fed the diets containing DSCF at various concentrations, proposing that DSCF supplementation did not produce morphological and functional alterations in the fish intestines.

The intestinal epithelium comprises a single layer of enterocytes with an apical brush border (Lazo et al., 2011). The physiological functions of enterocytes are responsible for nutrient uptake and ion transport processes (Wilson and Castro, 2011). Mucus secretion from goblet cells distributed throughout the gastrointestinal tract of fish, which plays a key role in 1) the regulation of digestion and absorption of the chyme; 2) the protection of the intestinal mucosa from mechanical and chemical digestion processes; and 3) the protection of fish from the number of pathogenic bacteria that may exert harmful effects on health and well-being (Lazo et al., 2011; Wilson and Castro, 2011). Observations on micromorphology of the intestine are useful for the interpretation of the 
nutritional conditions in fish. In this study, it was observed that intestinal epithelial cells are arranged throughout the villi compactly and each enterocyte is continually lined by microvilli at the apical surface. The sizes of enterocyte nuclei varied and can be categorized into 2 subtypes; supranucleus and subnucleus (Escaffre et al., 2007). It was postulated that supranucleus height, subnucleus height, nucleus height, and nucleus width are cellular criteria used to evaluate the nutritional conditions in fish (Lazo et al., 2011; Ferrara et al., 2015). In the present study, epithelial abrasion, cellular necrosis, and inflammatory signs were not found in all specimens of fish fed the experimental diets compared with the control. Increased enterocyte height was noticed in fish fed with the diets incorporated with DSCF at the levels of 1 and 3\%, whereas the highest dose of DSCF (5\%) caused a significant decrease in enterocyte height. It has been reported that increased microvilli and enterocyte height would support nutrient assimilation in the tract of fish (Munglue et al., 2019). The reduction in the enterocyte height is prominently correlated with suboptimal feeding or starvation in fish (Ostaszewska et al., 2005; Lazo et al., 2011). As mentioned above, decreased enterocyte height was observed in fish fed the diet supplemented with $5 \% \mathrm{DSCF}$ and this morphological change did not affect somatic growth in the fish. The mechanism by which dietary administration of DSCF reduced enterocyte height is not clear. It was indicated that diet compositions and environmental variations could primarily reflect cell metabolism and cell integrity in the fish intestines (Wilson and Castro, 2011; Munglue et al., 2019). A previous report has indicated that catfish fed the diets supplemented with L. aromatica extract at a high level showed a significant decrease in enterocyte height (Munglue et al., 2019). In this current study, an increase in supranucleus height, subnucleus height, nucleus height, and nucleus width in the intestines of fish fed the diets containing 1 and 3\%DSCF could be graded the intestinal epithelium as 'healthy tissues' (Lazo et al., 2011). Therefore, these findings suggest that the application of DSCF to the diet should not more than $3 \%$ in order to reduce micromorphological alterations of the intestines.

Hematological parameters are useful for the indication of health status and nutritional assimilation of fish (Tiengtam et al., 2015; Sewaka et al., 2019). In general, measurement values of blood indices recorded in the present study are within the acceptable limits for hybrid catfish (Al-Dohail et al., 2009). In this current research, no changes in $\mathrm{RBC}, \mathrm{Hb}$, and Hct levels were noticed in the tested fish compared with the control. However, WBC and platelet count were significantly increased in fish fed 5\%DSCF containing diet. It was demonstrated that such phytochemicals could activate hematopoietic tissues to produce a high level of WBC and platelet in fish (Gabriel et al., 2015; Abdel-Tawwab et al., 2018; Mohammadi et al., 2018).

Blood biochemical parameters have been used to evaluate physiological responses of fish that were fed with the diets containing herbal plant extracts (Gabriel et al., 2015; Ahmadifar et al., 2019; Sewaka et al., 2019). This present 
finding exhibited the highest level of glucose was observed in fish fed the diet containing 5\%DSCF compared with the control. It has been reported that a rise in blood glucose might be attributed to an increase in energy demand in response to physiological stress in the cultured fish (Mutlu et al., 2015). Therefore, it is considered that dietary supplementation with 5\%DSCF may induce stress in fish. AST and ALP levels are directly correlated with liver functions (Tiengtam et al., 2015). Increased AST and ALP contents may indicate hepatic disorders (Hassaan et al., 2019). Decreased AST and ALP levels would demonstrate the hepatoprotective effects of the tested phytogenic additive in fish (Gabriel et al., 2015). In this research, fish that were fed with $1 \%$ DSCF diet showed a significant decrease in serum ALP, suggesting that $S$. cumini was effective in preventing the hepatocyte damage in fish (Baliga et al., 2011). Serum total protein, albumin, creatinine, and uric acid are generally used to evaluate the health status of the gill, muscle, liver, and kidney (Gabriel et al., 2015; Mutlu et al., 2015). Increased levels of total protein, albumin, creatinine, triglyceride, and uric acid are associated with stress, liver and kidney diseases, gill disorders, and metabolic syndrome (Gabriel et al., 2015; Mutlu et al., 2015; Tiengtam et al., 2015). In this study, the levels of AST, albumin, total protein, creatinine, uric acid, and triglyceride were similar in all treatments. Thus, it is suggested that dietary DSCF did not have any adverse effects on the metabolic processes and the health status of the liver and kidney of fish (Gabriel et al., 2015; Hassaan et al., 2019).

\section{CONCLUSION}

In summary, the results of the present study demonstrated that DSCF could be used as a phytogenic additive in the diets in order to improve growth performance, intestinal morphology, hematology, and blood biochemistry in hybrid catfish. By using the second-order polynomial analysis, the optimal level of DSCF was determined to be between 2.60 and $2.71 \% / \mathrm{kg}$ diet.

\section{ACKNOWLEDGMENTS}

The authors would like to thank the Faculty of Science, Ubon Ratchathani Rajabhat University for providing instruments. The authors are grateful to $\mathrm{Mr}$ Kittanai Krongyoot and Miss Phatcharee Bunthao for data collection during the experimental period.

\section{REFERENCES}

Aanyu, M., Betancor, M.B., and Monroig, O. 2018. Effects of dietary limonene and thymol on growth and nutritional physiology of Nile tilapia (Oreochromis niloticus). Aquaculture. 488: 217-226. https://doi.org/10. 1016/j.aquaculture.2018.01.036 
Abdel-Tawwab, M., Adeshina, I., Jenyo-Oni, A., Ajani, E.K., and Emikpe, B.O. 2018. Growth, physiological, antioxidant, and immune response of African catfish, Clarias gariepinus (B.), to dietary clove basil, Ocimum gratissimum, leaf extract and its susceptibility to Listeria monocytogenes inflection. Fish and Shellfish Immunology. 78: 346-354. https://doi.org/ 10.1016/j.fsi.2018.04.057

Ahmadifar, E., Sheikhzadeh, N., Roshanaei, K., Dargahi, N., and Faggio, C. 2019. Can dietary ginger (Zingiber officinale) alter biochemical and immunological parameters and gene expression related to growth, immunity and antioxidant system in zebrafish (Danio rerio)? Aquaculture. 507: 341-348. https://doi.org/10.1016/j.aquaculture. 2019.04.049

Al-Dohail, M.A., Hashim, R., and Aliyu-Paiko, M. 2009. Effects of the probiotic, Lactobacillus acidophilus, on the growth performance, haematology parameters and immunoglobulin concentration in African Catfish (Clarias gariepinus, Burchell 1822) fingerling. Aquaculture Research. 40(14): 1642-1652. https://doi.org/10.1111/j.1365-2109.2009.02265.x

Aqil, F., Gupta, A., Munagala, R., Jeyabalan, J., Kausar, H., Sharma, R.J., Singh, I.P., and Gupta, R.C. 2012. Antioxidant and antiproliferative activities of anthocyanin/ellagitannin-enriched extracts from Syzygium cumini L. (Jamun, the Indian blackberry). Nutrition and Cancer. 64(3): 428-438. https://doi.org/10.1080/ 01635581.2012.657766

Awad, E., and Awaad, A. 2017. Role of medicinal plants on growth performance and immune status in fish. Fish \& Shellfish Immunology. 67: 40-54. https://doi.org/10.1016/j.fsi.2017.05.034

Ayyanar, M., and Subash-Babu, P. 2012. Syzygium cumini (L.) skeels: a review of its phytochemical constituents and traditional uses. Asian Pacific Journal of Tropical Biomedicine. 2(3): 240-246. https://doi.org/10.1016/ S2221-1691(12)60050-1

Baeverfjord, G., and Krogdahl, A. 1996. Development and regression of soybean meal induced enteritis in Atlantic salmon, Salmo salar L., distal intestine: a comparison with the intestines of fasted fish. Journal of Fish Disease. 19: 375-387. https://doi.org/10.1111/j.1365-2761.1996.tb00376.x

Baliga, M.S., Bhat, H.P., Baliga, B.R.V, Wilson, R., and Palatty, P.L. 2011. Phytochemistry, traditional uses and pharmacology of Eugenia jambolana Lam. (black plum): a review. Food Research International. 44(7): 17761789. https://doi.org/10.1016/j. foodres.2011.02.007

Bunting, S.W. 2013. Principles of sustainable aquaculture. Routledge. U.S.A.

Campbell, T.W., and Ellis, C.K. 2007. Avian and exotic animal hematology and cytology $3^{\text {rd }}$ ed. U.S.A.: Wiley-Blackwell. https://doi.org/10.1111/j.17510813.2007.00165.x 
Dekić, R., Savić, N., Manojlović, M., Golub, D., and Pavličević, J. 2016. Condition factor and organosomatic indices of rainbow trout (Oncorhynchus mykiss) from different broad stock. Biotechnology in Animal Husbandry. 32(2): 229-237. https://doi.org/10.2298/BAH160222 9D

Escaffre, A.M., Kaushika, S., and Mambrinib, B. 2007. Morphometric evaluation of changes in the digestive tract of rainbow trout (Oncorhynchus mykiss) due to fish meal replacement with soy protein concentrate. Aquaculture. 273(1): 127-138. https://doi.org/10.1016/j.aquaculture.2007.09.028

Evans, W.C., Evans, D., and Trease, G.E. 2002. Trease and Evans pharmacognosy. $15^{\text {th }}$ ed. Edinburgh. U.K.: Saunders/Elsevier.

Food and Agriculture Organization of the United Nations, Fisheries and Aquaculture Department (FAO.). 2012. The state of world fisheries and aquaculture. Food and Agriculture Organization of the United Nations. Rome; London: Eurospan.

Ferrara, E., Gustinelli, A., Fioravanti, M.L., Restucci, R., Quaglio, F., Marono, S., and Piccolo, G. 2015. Histological and micro-/macromorphological evaluation of intestine in sharpsnout seabream (Diplodus puntazzo) fed soybean meal-based diets added with MOS and inulin as prebiotics. Aquaculture International. 23(6): 1525-1537.https://doi.org/ 10.1007/s10499-015-9902-y

Gabriel, N.N., Qiang, J., He, J., Ma, X.Y., Kpundeh, M.D., and Xu, P. 2015. Dietary Aloe vera supplementation on growth performance, some haematobiochemical parameters and disease resistance against Streptococcus iniae in tilapia (GIFT). Fish \& Shellfish Immunology. 44: 504-514. https://doi. org/10.1016/j.fsi.2015.03.002

Gabriel, N.N., Qiang, J., Ma, X.Y., He, J., Xu, P., and Liu, K. 2015. Dietary Aloe vera improves plasma lipid profile, antioxidant, and hepatoprotective enzyme activities in GIFT-tilapia (Oreochromis niloticus) after Streptococcus iniae challenge. Fish Physiology and Biochemistry. 41(5): 1321-1332. https://doi.org/10.1007/s10695-015-0088-Z

Gabriel, N.N., Wilhelm, M.R., Habte-Tsion, H.M., Chimwamurombe, P., and Omoregie, E. 2019. Dietary garlic (Allium sativum) crude polysaccharides supplementation on growth, haematological parameters, whole body composition and survival at low water $\mathrm{pH}$ challenge in African catfish (Clarias gariepinus) juveniles. Scientific African. 5: e00128. https://doi. org/10.1016/j.sciaf.2019.e00128

Gupta, A., and Sandhu, R.S. 1998. Effect of garlic agglutinin and garlic extracts on the rat jejunum. Nutritional Research. 18(5): 841-850. https://doi.org/ 10.1016/S0271-5317(98)00069-4

Gupta, M.V. 2006. Challenges in sustaining and increasing fish production to combat hunger and poverty in Asia. Naga World Fish Center Quarterly. 29(1\&2): 4-10. 
Haghbayan, S., and Mehrgan, M.S. 2015. The effect of replacing fish meal in the diet with enzyme-treated soybean meal (HP310) on growth and body composition of rainbow trout fry. Molecules. 20: 21058-21066. https://doi. org/10.3390/molecules201219751

Hassaan, M.S., Mohammady, E.Y., Soaudy, M.R., El-Garhy, H.A.S., Moustafa, M.M.A., Mohamed, S.A., and El-Haroun, E.R. 2019. Effect of Silybum marianum seeds as a feed additive on growth performance, serum biochemical indices, antioxidant status, and gene expression of Nile tilapia, Oreochromis niloticus (L.) fingerlings. Aquaculture. 509: 178-187. https://doi.org/10.1016/j.aquaculture.2019.05.006

Lazo, J.P., Darias, M.J., and Gisbert, E. 2011. Ontogeny of the digestive tract. In: Holt, G.L., editor. Larval Fish Nutrition UK: John Wiley \& Sons, Inc. p.546. https://doi.org/10.1002/9780470959862.ch1

Mohamed, A.A., Ali, S. I., and El-Baz, F.K. 2013. Antioxidant and antibacterial activities of crude extracts and essential oils of Syzygium cumini leaves. Plos One. 8(4): e60269. https://doi.org/10.1371/journal.pone.0060269

Mohammadi, M., Soltani, M., Siahpoosh, A., Shekarabi, S.P.H., Mehrgan, M.S., and Lymbery, A. 2018. Effect of date palm (Phoenix dactylifera) seed extract as a dietary supplementation on growth performance immunological haematological biochemical parameters of common carp. Aquaculture Research. 49(8): 1-10. https://doi.org/ 10.1111/are.13760

Munglue, P., Rattana, K., Sangchanjiradet, S., and Dasri, K. 2019. Effect of dietary lasia (Lasia spinosa (L.) Thwaites) extract on growth performance and intestinal histology in hybrid catfish (Clarias macrocephalus $\times$ Clarias gariepinus). Chiang Mai University Journal of Natural Sciences. 18(2): 226-249. https://doi.org/10.12982/ CMUJNS.2019.0017

Munglue, P., Rattana, K., Sangchanjiradet, S., Jankam, A., and Dasri, K. 2019. Growth performance and intestinal morphology of hybrid catfish (Clarias macrocephalus $\times$ Clarias gariepinus) fed diet supplemented with rice paddy herb (Limnophila aromatica) extract. Asia-Pacific Journal of Science and Technology. 24(2): 1-12.https://doi.org/10.14456/apst.2019. 17

Mutlu, E., Aydın, S., and Kutlu, B. 2015. Alterations of growth performance and blood chemistry in Nile tilapia (Oreochromis nioticus) affected by copper sulfate in long-term exposure. Turkish Journal of Fisheries and Aquatic Sciences. 15: 487-493. https://doi.org/10.4194/1303-2712-v15_2_35

Ostaszewska, T., Dabrowski, K., Palacios, M.E., Olejniczak, M., and Wieczorek, M. 2005. Growth and morphological changes in the digestive tract of rainbow trout (Oncorhynchus mykiss) and pacu (Piaractus mesopotamicus) due to casein replacement with soybean proteins. Aquaculture. 245(1-4): 273-286. https://doi.org/ 10.1016/j.aquaculture. 2004.12.005 
Panase, P., Kamee, B., Moungmor, S., Tipdacho, P., Matidtor, J.B., and Sutthi, N. 2018. Effects of Euphorbia hirta plant leaf extract on growth performance, hematological and organosomatic indices of hybrid catfish, Clarias macrocephatus $\times$ C. gariepinus. Fisheries Science. 84: 10251036. https://doi.org/10.1007/s12562-018-1234-1

Prabu, D.L., Chandrasekar, S., Ambashankar, K., Dayal, J.S., Ebeneezar, S., Ramachandran, K., Kavitha, M., and Vijayagopal, P. 2018. Effect of dietary Syzygium cumini leaf powder on growth and non-specific immunity of Litopenaeus vannamei (Boone 1931) and defense against virulent strain of Vibrio parahaemolyticus. Aquaculture. 489: 9-20. https://doi.org/10. 1016/j.aquaculture.2018.01.041

Reverter, M., Bontemps, N., Lecchini, D., Banaigs, B., and Sasal, P. 2014. Use of plant extracts in fish aquaculture as an alternative to chemotherapy: current status and future perspectives. Aquaculture. 433: 50-61. https:// doi.org/10.1016/j.aquaculture. 2014.05.048

Robinson, E.H., Li, M.H., and Manning, B.B. 2001. A practical guide to nutrition, feeds, and feeding of catfish (second revision). Mississippi: Office of Agricultural Communications.

Serrano, E., Storebakken, T., Borquez, A., Penn, M., Shearer, K.D., Dantagnan, P., and Mydland, L.T. 2012. Histology and growth performance in rainbow trout (Oncorhynchus mykiss) in response to increasing dietary concentration of sparteine, a common alkaloid in lupins. Aquaculture Nutrition. 18: 313-320. https://doi.org/10.1111/j.1365-2095.2011.00899.x

Sewaka, M., Trullas, C., Chotiko, A., Rodkhum, C., Chansue, N., Boonanuntanasarn, S., and Pirarat, N. 2019. Efficacy of symbiotic Jerusalem artichoke and Lactobacillus rhamnosus GG-supplemented diets on growth performance, serum biochemical parameters, intestinal morphology, immune parameters and protection against Aeromonas veronii in juvenile red tilapia (Oreochromis spp.). Fish and Shellfish Immunology. 86: 260-268. https://doi.org/10.1016/j.fsi.2018.11.026

Sruthi, M.V., Nair, A.B., Arun, D., Thushara, V.V., Sheera, C.C., Vijayasree, A.S., Oommen, O.V., and Divya, L. 2018. Dietary curcumin influences leptin, growth hormone and hepatic growth factors in tilapia (Oreochromis mossambicus). Aquaculture. 496: 105-111. https://doi.org/10.1016/ j.aquaculture.2018.06.083

Tiengtam, N., Khempaka, S., Paengkoum, P., and Boonanuntanasarn, S. 2015. Effects of inulin and Jerusalem artichoke (Helianthus tuberosus) as prebiotic ingredients in the diet of juvenile Nile tilapia (Oreochromis niloticus). Animal Feed Science and Technology. 207: 120-129. https://doi.org/10.1016/j.anifeedsci.2015.05.008 
Villasante, A., Powell, M.S., Moutou, K., Murdoch, G.K., Overturf, K., Wacyk, J., and Hardy, R.W. 2016. Effects of anthocyanidins on myogenic differentiation and antioxidant defense in primary myogenic cells isolated from rainbow trout (Oncorhynchus mykiss). Aquaculture. 454: 81-89. https://doi.org/10.1016/j. aquaculture.2015.12.007

Wedemeyer, G.A. 1996. Physiology of fish in intensive culture systems. New York: Chapman and Hall. https://doi.org/10.1007/978-1-4615-6011-1

Wilson, J.M., and Castro, L.F.C. 2011. Morphological diversity of the gastrointestinal tract in fishes. In: Grosell, M., Farrell, A.P., and Brauner, C.J., editors. the Multifunctional Gut of Fish p. 1-55. London: Academic Press. https://doi.org/10.1016/S1546-5098(10)03001-3 